Check for updates

Cite this: RSC Adv., 2019, 9, 24638

\title{
Fluorescent perylene derivative functionalized titanium oxide gel for sensitive and portable ascorbic acid detection $\uparrow$
}

\author{
Binbin Zhang, Wenxia Liu, Yihao Liu, Zhiguang Suo, Lingyan Feng, (D) * Feifei Xing* \\ and Shourong Zhu ID
}

An inorganic titanium oxide ( $\mathrm{TiO}$ ) gel sensor was demonstrated for convenient detection of ascorbic acid (AA). It is composed of $\mathrm{TiO}(\mathrm{PI}-\mathrm{TiO})$ functionalized with a perylene diimide derivative containing carboxylic groups as a new soft dopant material. A traditional solvothermal reaction is adopted to prepare the $\mathrm{PI}-\mathrm{TiO}$ composite, which exhibits a different spectrum according to the reaction time. The final gel possesses a strong chelating affinity with AA molecules, in which phenol hydroxyl groups are shown to compete with those already present in PI. We further utilize the functionalized gel to prepare a series of films with a simple and portable AA response. A visual colour variation can be recognized by the naked eye, together with obvious fluorescence changes for selective and sensitive AA detection. Finally, the as-prepared gel film displays good stability and reproducibility for real sample responses with satisfying results.

Received 4th March 2019

Accepted 30th June 2019

DOI: $10.1039 / c 9 r a 01621 j$

rsc.li/rsc-advances

perylene compounds at the imide or bay positions, increasing the whole molecular solubility. ${ }^{\mathbf{1 2 - 1 5}}$ Due to the presence of carboxyl substituents, interactions between PI and TiO are expected, making a hybrid composite with unique optical properties. ${ }^{8}$ However, few works have reported entrapping PI dyes in TiO gels, and still no application has been discussed to utilize the interesting phenomenon of a fluorescent functionalized gel, especially for sensor development.

Ascorbic acid (AA) is a necessary compound in maintaining normal growth of the human body. It has important influence on many aspects of life activities, such as a lower risk of cardiovascular disease, stroke and cancer, and increased longevity. ${ }^{16}$ Traditional methods for AA detection include redox titration, HPLC, ${ }^{17}$ electrochemical detection ${ }^{18}$ and so on. Different methods have their strengths, but also some limitations. For example, the redox titration method to determine the chromogenic reaction is often slow, unstable and lacks sensitivity; the error of test results is relatively large. ${ }^{19}$ HPLC and electrochemical methods are usually sensitive enough, but they often need expensive instruments, as well as tedious experimental procedures. ${ }^{\text {20-22 }}$ Compared with those methods, optical detection has several advantages, which are high sensitivity and convenience, and that direct colour changes in some systems can be visually observed by the naked eye. It is also vital to design portable AA sensors for potential point-of-care testing (POCT) uses. $^{23}$

Here we develop one novel PI-TiO gel sensor for highly sensitive and selective AA detection, the principle for which is based on the stronger interaction between TiO and contiguous phenol hydroxyl groups in AA than with the -COOH groups,
Department of Chemistry, College of Science, Materials Genome Institute, Shanghai University, Shanghai 200444, China

$\dagger$ Electronic supplementary information (ESI) available. See DOI: 10.1039/c9ra01621j 


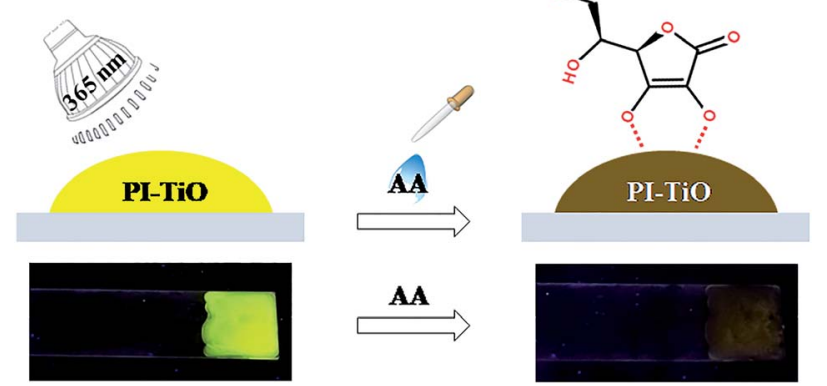

Scheme 1 Schematic diagram of PI-TiO gel on glass for AA detection using a portable UV lamp.

quenching PI fluorescence efficiently. The inorganic gel-based biosensor is also portable, prepared on simple glass substrate, and so visual colour and fluorescence changes can be obtained conveniently. The detection process is shown in Scheme 1. The prepared PI-TiO gel was easily spread on a clean glass substrate, with high FL emission under a $365 \mathrm{~nm}$ ultraviolet lamp. When AA solution was dropped onto the gel surface, the optical intensity immediately decreased depending on the AA concentration. The optical change was ascribed to the competitive binding of AA with titanium which promotes the disassembly of the PI-TiO complex. Controlled experiments demonstrate that the established gel method has high sensitivity and selectivity for AA, different from other analogous molecules. Since the $365 \mathrm{~nm}$ lamp is inexpensive compared with other complicated instruments, the gel substrate is also stable enough for portable applications.

\section{Experimental}

\subsection{Chemicals and characterization}

All analytically pure reagents, $\mathrm{Ti}\left\{\mathrm{OCH}\left(\mathrm{CH}_{3}\right)_{2}\right\}_{4}(99 \%), 3,4,9,10-$ perylenetetracarboxylic dianhydride (PTCDA), 5-aminoisophthalic acid (98\%), imidazole (ACS reagent, 99\%), etc., and solvents were purchased from commercial sources and used without further purification. ${ }^{1} \mathrm{H}$ NMR spectra were carried out using a Bruker AVANCE $500 \mathrm{MHz}$ spectrometer. Emission and absorption spectra were collected using a PERSEE TU-1950 UVvisible spectrophotometer and an Edinburgh Instruments FS5 fluorescence spectrometer. The fluorescence light source was provided by $150 \mathrm{~W}$ xenon lamp and the fluorescence signal was amplified by a R982P photomultiplier tube. $360 \mathrm{~nm}$ was selected as the excitation wavelength, and the scan slit was $1.5 \mathrm{~nm}$. Step was set at $2 \mathrm{~nm}$ and wavelength accuracy is $\pm 0.5 \mathrm{~nm}$. The detector sensitivity is $>6000: 1$. FT-IR spectra were observed as $\mathrm{KBr}$ pellets on a Thermo Fisher Nicolet is50 spectrometer. The morphologies of the resulting films were investigated using scanning electron microscopy (SEM, ZEISS Sigma500). Room temperature X-ray diffraction data were obtained using a Bruker D2 phaser desktop X-ray diffractometer. Thermal analysis was conducted using a NETZSCH STA 2500 Regulus simultaneous thermal analyzer. The samples were heated under a nitrogen stream of $20 \mathrm{~mL} \mathrm{~min}^{-1}$, with a heating rate of $20{ }^{\circ} \mathrm{C} \mathrm{min}^{-1}$. X-ray photoelectron spectroscopy (XPS) measurements were recorded using a Thermo Fisher Scientific WSCALAB spectrometer.

\subsection{Synthesis of perylene diimide derivative (PI)}

The perylene diimide derivative with four carboxylic acid groups (PI) was synthesized as reported previously. ${ }^{24}$ Briefly, PTCDA $(0.50 \mathrm{~g}), 5$-aminoisophthalic acid $(0.51 \mathrm{~g})$ and imidazole $(5.00 \mathrm{~g})$ were heated at $130{ }^{\circ} \mathrm{C}$ for 12 hours under a nitrogen atmosphere. Then, the deep red solution was acidified with $2 \mathrm{M} \mathrm{HCl}$ to about $\mathrm{pH}=2$, and a red precipitate was obtained. Centrifugation, water washing and ethanol washing were performed three times, after that the product was dried at $80{ }^{\circ} \mathrm{C}$ in a vacuum oven to obtain $0.916 \mathrm{~g}$ of deep red powder (yield: $91.8 \%)$.

\subsection{Synthesis of the PI-TiO composite}

The PI-TiO composite was prepared from titanium isopropoxide and a minute quantity of PI in ethanol by a solvothermal method. In a typical procedure, $2.0 \mathrm{~mL}$ of titanium tetraisopropanolate $\left(\mathrm{Ti}(\mathrm{OR})_{4}\right)$ was dissolved in $4.0 \mathrm{~mL}$ of ethanol at room temperature, $1.0 \mathrm{mg}$ of PI was added, and then the mixture was stirred then transferred into a $10 \mathrm{~mL}$ Teflon-lined autoclave at $40{ }^{\circ} \mathrm{C}$ for 6 hours (alterable). The resulting solutions were purified by filtration to remove tiny insoluble impurities, giving a clear solution.

\subsection{Synthesis of the PI-TiO gel}

In order to control the reaction, the target product was prepared by a typical sol-gel method, and a PI-TiO solution was used as a precursor. Acetic acid $(0.05 \mathrm{~mL}, 98 \%)$ was added to the PI-TiO solution $(5 \mathrm{~mL})$ at a constant stirring rate at room temperature. Deionized water $(0.075 \mathrm{~mL})$ was added drop-by-drop with continuous stirring, and $\mathrm{pH}$ was adjusted to 3 by HCl. PI-TiO gel was obtained after letting it rest at room temperature overnight. To prepare the PI-TiO film on glass, fresh PI-TiO sol was directly transferred to a glass dropper and dripped onto a pretreated clean glass sheet. Under normal barometric pressure, ambient temperature $20{ }^{\circ} \mathrm{C}$ and humidity $\sim 60 \%$, the film was evaporated.

\section{Results and discussion}

\subsection{Preparation of the PI-TiO solution and gel}

PI with four carboxylic acid groups was synthesized as described in the Experimental section. ${ }^{25}$ Scheme 2 shows the chemical structure of PI and the solvothermal synthesis of the PI-TiO solution. PI can be dissolved easily in the $\mathrm{Ti}(\mathrm{OR})_{4}$-ethanol solution by gentle thermal treatment, since the acidolysis reaction occurs as previously reported. ${ }^{26,27}$

At first, the colour of the dissolved solution is light yellow. Once the solvothermal reaction time has passed, the colour turns a dark yellow (Fig. 1a). After different lengths of time $(1.5 \mathrm{~h}, 6.0 \mathrm{~h}$ and $24 \mathrm{~h})$, the colour appears quite different without an obvious relationship. Time varies the colour of the 
(a)

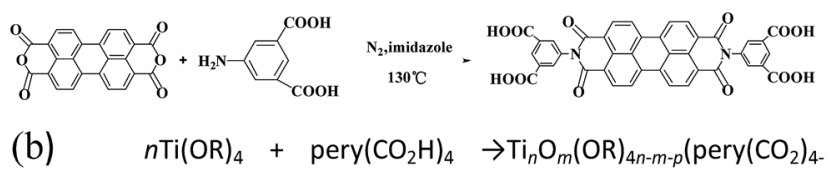

$\left.{ }_{m}(\mathrm{COOR})_{m}\right)+p \mathrm{HOR}$

Scheme 2 (a) Synthesis and chemical structure of PI and (b) synthesis of $\mathrm{PI}-\mathrm{TiO}$ solution

solution due to the reaction of different quantities of PI and $\mathrm{Ti}(\mathrm{OR})_{4}$. The corresponding fluorescence colours of the PI-TiO solution are obviously green, orange and yellow; the latter with a $24 \mathrm{~h}$ reaction time possesses the brightest fluorescence performance (Fig. 1b). A transparent PI-TiO gel was further obtained from the PI-TiO solution using a sol-gel method at $40{ }^{\circ} \mathrm{C} .{ }^{28}$ By carefully adjusting the $\mathrm{pH}$ value to 3 , it became a glue-like state at room temperature overnight. As shown in Fig. 1c, we can obtain different PI-TiO gels at the corresponding reaction times in solution. The whole gel is green after $1.5 \mathrm{~h}$ reaction time, a brown-yellow gel forms for the $6 \mathrm{~h}$ solution, and finally a bright yellow PI-TiO gel was obtained for the $24 \mathrm{~h}$ solution. On aging, the PI-TiO gel slowly becomes a dry, reddish glue to the naked eye with the volatilization of the solvent (Fig. 1d).

The colour changes and different fluorescent emissions were due to the PI molecule assembly behavior along with the reaction time, as shown in Fig. 1e. At the beginning of the reaction, the peak is strong around $530 \mathrm{~nm}$ and the form of perylene monomer is green. With an increase in reaction time, the
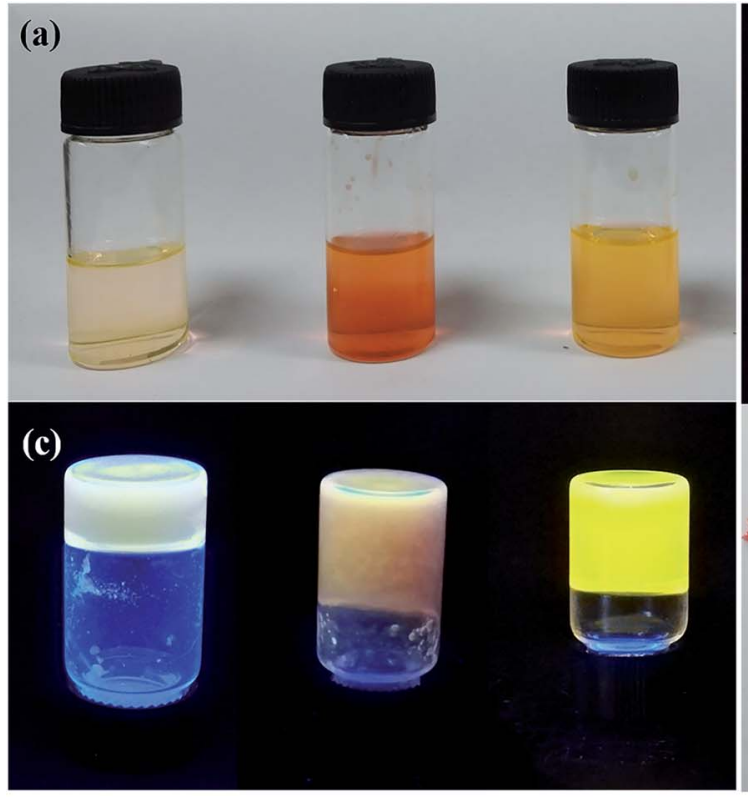

emergence of a new peak at $580 \mathrm{~nm}$ is more obvious. Perylene derivatives have colour changes and fluorescence changes ascribed to the continuous aggregation of molecules. ${ }^{29,30}$ The aggregated state of molecules will form as the reaction time increases due to the interaction between molecules, finally, yellow fluorescence appears after $24 \mathrm{~h}$ reaction time. ${ }^{6,8}$

\subsection{Characterization of the PI-TiO gel}

We further conducted detailed characterization of the asprepared PI-TiO gel. Ti, C and O elements were found in the gel powder via EDS analysis, and their content is shown in Fig. 2a. Because hydrochloric acid is used during gelation, there is also a very small amount of $\mathrm{Cl}$ element. The SEM diagram of the gel powder shows the surface morphology of the aggregated TiO nanoparticles (Fig. 2b-c). XRD results (Fig. S1†) for the PITiO gel show that the gel is amorphous, and the two weak peaks at (101) and (004) are ascribed to anatase peaks of TiO. Further XPS results were obtained to demonstrate the valence bond states, as shown in Fig. $2 \mathrm{~d}-\mathrm{f}$. The abscissa is binding energy (eV) and the ordinate is relative intensity. The peaks at $458.1 \mathrm{eV}$ and $463.9 \mathrm{eV}$ correspond to $\mathrm{Ti} 2 \mathrm{p}_{3 / 2}$ and $\mathrm{Ti} 2 \mathrm{p}_{1 / 2}$ in TiO, respectively (Fig. 2d), while the peak at $529.6 \mathrm{eV}$ corresponds to oxygen in the TiO composite (Fig. 2e). The conjugated carbon of the perylene was also clearly detected at $284.2 \mathrm{eV}$, and the carboxyl carbon is displayed at $288.4 \mathrm{eV}$ (Fig. 2f). ${ }^{31,32}$

The UV spectrum of the dry gel shows a broad absorption around $480-540 \mathrm{~nm}$, which proves the existence of perylene (Fig. 3a). Infrared (IR) spectroscopy can help us better understand the state of organic molecules doped in inorganic materials. Three different samples, pure TiO dry gel, PI and PI-TiO dry gel, were characterized using IR (Fig. $3 \mathrm{~b}$ ). The carboxylic

Fig. 1 Photographs of the PI-TiO solutions prepared over different times (left $1.5 \mathrm{~h}$, center $6 \mathrm{~h}$, right $24 \mathrm{~h}$ ) with different colours at (a) the naked eye and (b) fluorescence, (c) different fluorescence emissions of the corresponding gels, (d) the reddish dry glue after volatilization, and (e) timetraced normalized fluorescence spectra of the PI-TiO solution after $1.5 \mathrm{~h}$ (black line), $6 \mathrm{~h}$ (red line) and $24 \mathrm{~h}$ (yellow line). 


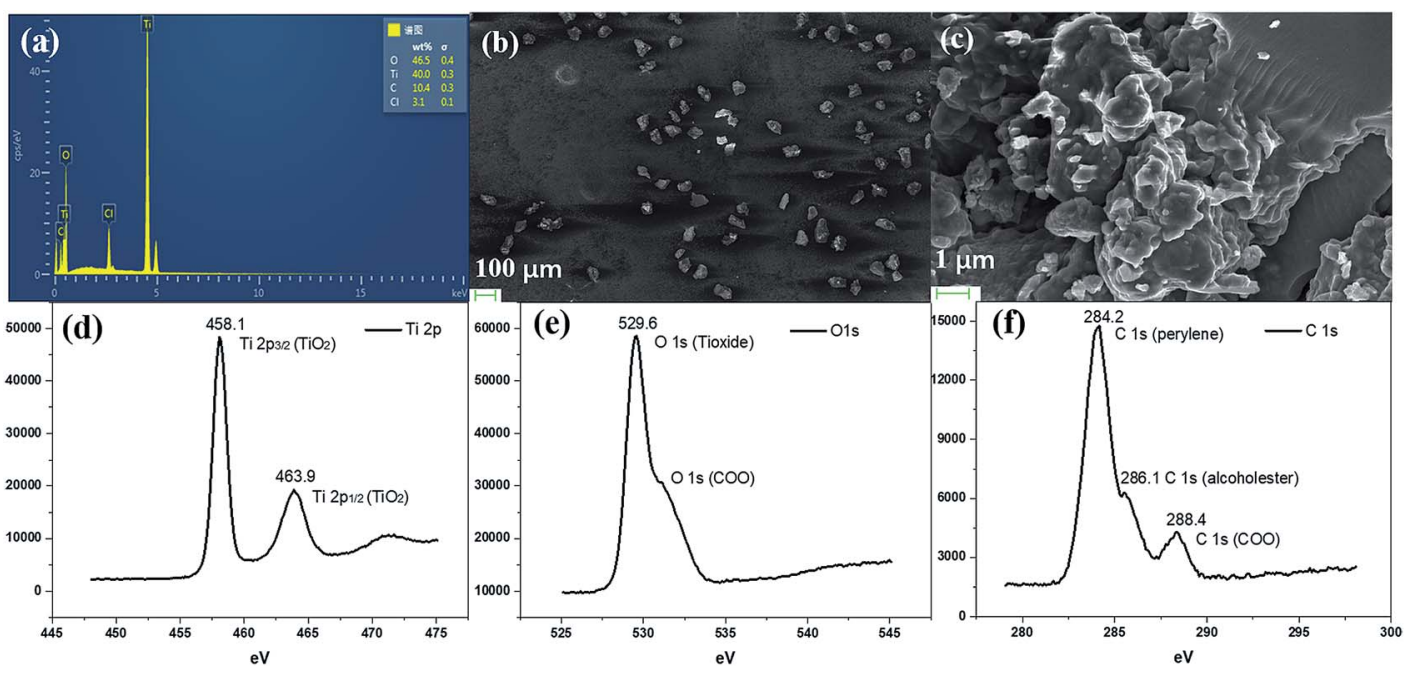

Fig. 2 (a) EDS analysis of the precipitate confirms that it contains Ti, O, C and Cl. (b and c) SEM images of the gel powder at different scales, and $(d-f)$ XPS results for the PI-TiO dry gel; (d) Ti 2p, (e) O 1s and (f) $C 1$ s.
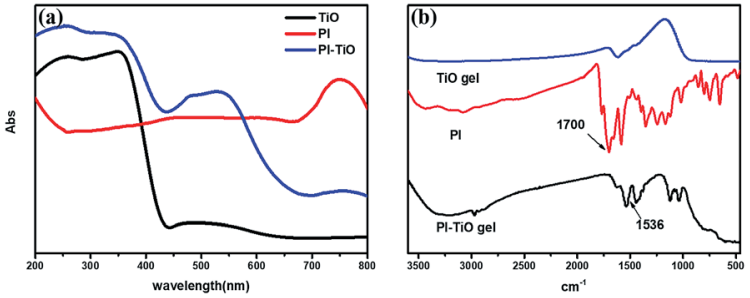

Fig. 3 UV-vis absorption (a) and FTIR spectra (b) of the pure TiO dry gel, $\mathrm{PI}$ and the PI-TiO dry gel.

acid peak of PI appears at $1700 \mathrm{~cm}^{-1}$ (red) and the peaks shift to about 1536 and $1438 \mathrm{~cm}^{-1}$ in the PI-TiO gel for the perylene ring $\nu_{\mathrm{C}=\mathrm{C}}$ and $\nu_{\mathrm{C}-\mathrm{N}}$ stretching vibrations, respectively (black). The red shift is due to the coordination of the metal-carboxylic group, meaning that the PI is chelated inside the PI-TiO gel. The formed Ti-O-C bond is indicated by the vibration peak around $1045 \mathrm{~cm}^{-1}$, between the $-\mathrm{COOH}$ and $\left.\mathrm{Ti}(\mathrm{Iv})\right)^{8,33}$ As mentioned in Scheme $2 \mathrm{~b}$, PI reacts with the Ti(OR $)_{4}$ agent, forming a PI-TiO compound.

Fig. S2 $\uparrow$ shows the thermogravimetric analysis (TG) curves for different degrees of solvent volatilization to form dry gel. The gel begins to lose solvent at room temperature, the curve drops sharply at $200{ }^{\circ} \mathrm{C}$, and then loses weight at about $380^{\circ} \mathrm{C}$. At this time, the dry gel gradually transforms into anatase. Because only a small amount of PTCA is input, the TG curves do not display an obvious perylene peak (benzene decomposition temperature is close to $600{ }^{\circ} \mathrm{C}$ ).

\subsection{Turn-off fluorescent detection of AA}

Although we can get three kinds of PI-TiO gels with different colors by adjusting the reaction time, the fluorescence of the green and orange gels is comparably weak. Therefore, we directly chose the yellow PI-TiO gel after $24 \mathrm{~h}$ reaction time in the subsequent AA tests. The absorption and fluorescence of
AA titration were firstly carried out using PI-TiO solution in quartz cuvettes. As illustrated in Fig. 4a, upon addition of AA in increased amounts, the absorbance of PI-TiO at the $490 \mathrm{~nm}$ and $525 \mathrm{~nm}$ peaks increased gradually. According to the literature ${ }^{34}$ perylene compounds are highly likely to aggregate due to the $\pi-\pi$ interactions of conjugated aromatic rings or intermolecular forces. The ratio of the absorption at 525 and $490 \mathrm{~nm}\left(A^{0-0} / A^{0-1}\right)$ indicates different aggregation states of perylene compounds. When $A^{0-0} / A^{0-1} \leq 0.7$, it exists mainly in the form of aggregates; when $A^{0-0} / A^{0-1}$ is approximately 1.6, only the monomer form dominates. The ratio varies between these two values according to the aggregation states. When the concentration of AA increases, $A^{0-0} / A^{0-1}$ of PI-TiO decreases gradually from 0.91 to 0.58 , indicating that AA concentration is conducive to the formation of PI aggregates. ${ }^{15}$ Correspondingly the fluorescence intensities at $542 \mathrm{~nm}$ and $578 \mathrm{~nm}$ were quenched linearly when AA was gradually added (Fig. 4b). Relative changes in fluorescence are more obvious than for UV measurements, so we then took the peaks around $542 \mathrm{~nm}$ for quantitative calculations. Fig. $\mathrm{S} 3 \uparrow$ presents the quantitative behavior of the portable PI-TiO luminescence quenching at $542 \mathrm{~nm}$. A detection limit of $18.0 \mu \mathrm{M}$ was calculated at $3 \sigma / S$, with a linear range between 0 and $0.2 \mathrm{mmol} \mathrm{L}^{-1} \mathrm{AA}\left(R^{2}=0.99\right)$. With the addition of AA amount, it was noticed that the PI-TiO colour gradually deepened from yellow to brown (Fig. 4c). Upon irradiation with the $365 \mathrm{~nm}$ UV lamp, the bright yellow fluorescence was slowly quenched under ultraviolet light in the presence of AA (Fig. $4 \mathrm{~d}$ ).

We further conducted solid fluorescence tests by fixing the gel on a solid holder to confirm the gel phenomenon changes (Fig. S4 $\dagger$ ). The location of the two peaks of the PI-TiO gel is like that of the previous solution peak. Once $2 \mu \mathrm{L}$ of AA solution (300 $\mu \mathrm{M}$ ) was dripped onto the gel with a pipette, the gel fluorescence intensity was quenched to less than half the original intensity. A second $2 \mu \mathrm{L}$ of the AA solution $(300 \mu \mathrm{M})$ caused the whole gel fluorescence to be almost quenched. Later, the gel was cast on 

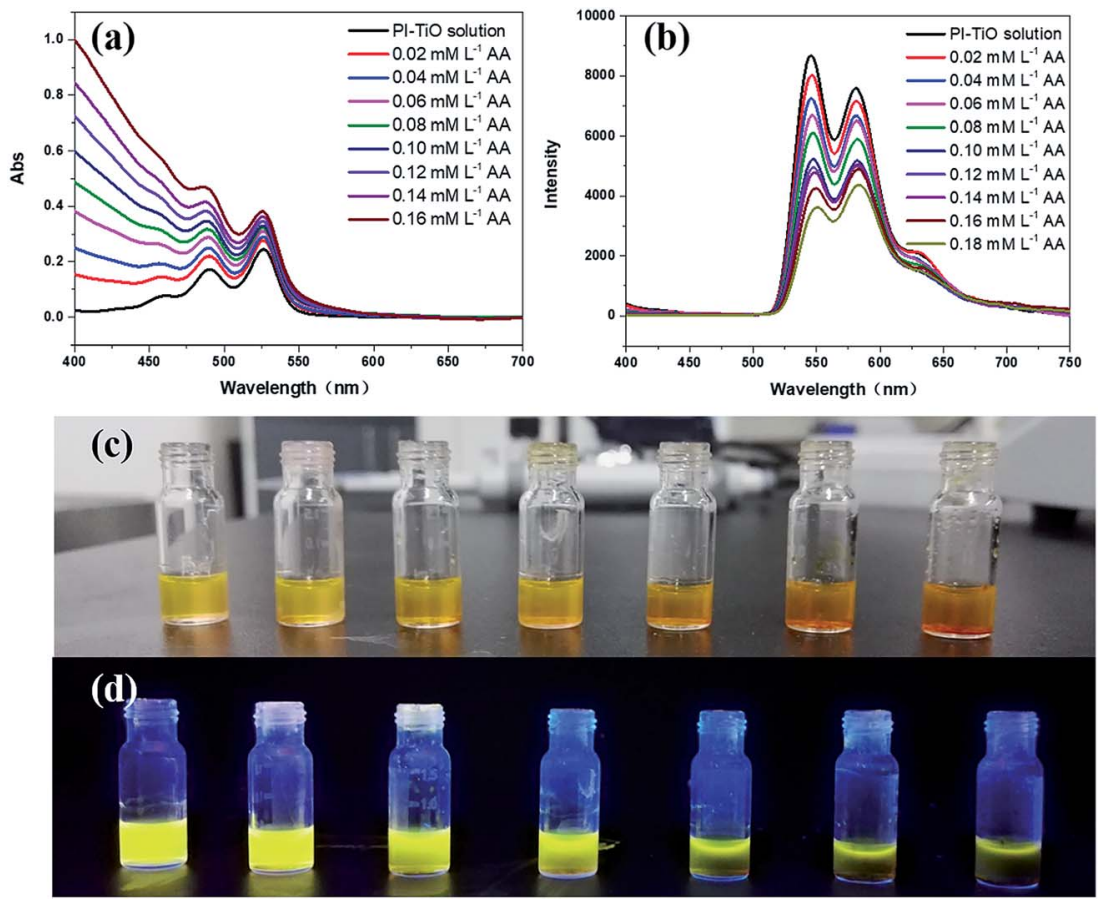

Fig. $4 \mathrm{UV} /$ vis absorption spectra (a), fluorescence spectra (Ex $=360 \mathrm{~nm}$, scan slit $=1.5 \mathrm{~nm}$ ) (b) and photographs of PI-TiO solution with AA solution gradually added (1.5 mM) under natural light (c) and under $365 \mathrm{~nm}$ light (d).

a glass plate to prepare a detection film (around $1 \mathrm{~cm}^{2}$ ). To the naked eye, the film is white, and a bright fluorescence emission of the gel film can be observed under a 365 UV lamp. Also, once the AA aqueous solution is dropped onto the film, the colour of the gel film changes from white to yellow, and the fluorescence emission is completely inhibited by the AA aqueous solution. These optical change phenomena on the film can be explained by the strong ligand transition of metal charge transfer. ${ }^{35}$ It is well known that $\mathrm{Ti}(\mathrm{Iv})$ can easily form complexes with oxygen atoms on various oxygen-containing ligands. ${ }^{36}$

\subsection{Detection principle and AA selectivity}

To fully prove the binding principle, we conducted various control experiments. As shown in Fig. 5, AA could completely inhibit the fluorescence emission of the PI-TiO films. Other organic acids at the same concentration, such as citric acid (CA), tartaric acid (TA) and oxalic acid (OA), have little effect on fluorescence emission. Also B12 vitamins and aromatic amines cannot quench the fluorescence emission of the PI-TiO film, as well as some other common amino acids. Interestingly, although the fluorescence of an aqueous solution of PI can be quenched by $\mathrm{Al}^{3+}, \mathrm{Cu}^{2+}$ and $\mathrm{Pb}^{2+}$, the PI-TiO obtained by a solgel method will not be affected by these, indicating a stronger binding ability of Ti(Iv) with PI. ${ }^{37}$ The glass substrate with PITiO gel on surface is stable for transfer during the experimental processes.

Selectivity is supposed to be due to the competitive coordination of PI and AA with TiO. Titanium in TiO can bind with AA to form ascorbic acid titanium (Ti(Iv)-AA), which is yellow (or reddish brown). The coordination between PI and titanium

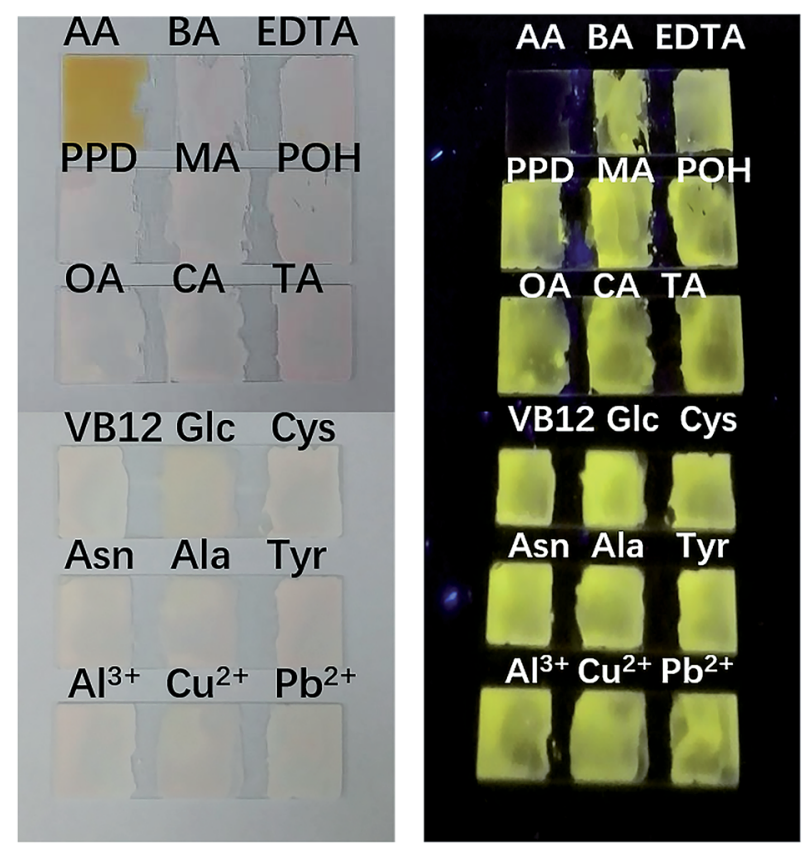

Fig. 5 Fluorescence response of the film to aqueous solutions of different compounds at the same concentration $(150 \mu \mathrm{M})$ (abbreviations: ascorbic acid, AA; benzoic acid, BA; ethylenediaminetetraacetic acid, EDTA; para-phenylenediamine, PPD; melamine, MA; phenol, $\mathrm{POH}$; oxalic acid, OA; citric acid, CA; tartaric acid, TA; vitamin B12, VB12; glucose, Glc; cysteine, Cys; aspartic acid, Asn; alanine, Ala; tyrosine, Tyr). 
oxide decreases, and the fluorescence of PI-TiO is destroyed by the new complex Ti(Iv)-AA. The bidentate binding of AA with anionic oxygen as an electron donor via two ene-diolate oxygen atoms is most likely because it results in a chelating ring structure, which provides increased stability. Furthermore, the five-membered ring structure has an advantageous angle configuration for atoms on the Ti surface, and the distortion of bond angle and distance is very small. ${ }^{38}$ The binding affinity increases with the phenolic hydroxyl groups in AA rather than the $-\mathrm{COOH}$ groups in PI. Also, the glycolic side chain of AA does not seem to be involved in the formation of complexes. ${ }^{35}$ An IR spectrogram was obtained to compare the characteristic absorption peaks of the PI-TiO gel before and after AA addition. As shown in Fig. $55 \dagger$ and $3 \mathrm{~b}$, the carboxylic acid peak of PI returned from $1536 \mathrm{~cm}^{-1}$ in PI-TiO to $1625 \mathrm{~cm}^{-1}$ after AA addition, indicating that the coordination of carboxylic groups with $\mathrm{Ti}(\mathrm{Iv})$ was successfully destroyed. Another new peak at $580 \mathrm{~cm}^{-1}$ confirmed the existence of Ti-O bonds, due to the coordination of Ti with hydroxyl groups in ascorbic acid..$^{38}$ It is said not all four of the carboxyl groups in PI were coordinated, and that some of these may be esterified. ${ }^{8}$

In order to further prove the coordination effect between phenolic hydroxyl groups and Ti(Iv), other compounds containing phenol hydroxyl groups, such as phenol, pyrocatechol and 1,2,3-benzenetriol solutions, were checked for their optical changes on PI-TiO gel film. As shown in Fig. 6, the fluorescence is also quenched by pyrocatechol and 1,2,3-benzenetriol solutions with a concentration higher than $300 \mu \mathrm{M}$, while phenol can also significantly weaken film fluorescence. When the concentration of phenol is upped to $2 \mathrm{mM}$, film fluorescence quenching occurs. In contrast, we dropped glucose and tartaric acid solutions which contain - $\mathrm{OH}$ on the gel film without any change in fluorescence. This is consistent with previous discussion and proves that the strong coordination of the hydroxyl group on the aromatic ring with Ti(Iv) causes colour change and fluorescence quenching. ${ }^{38}$

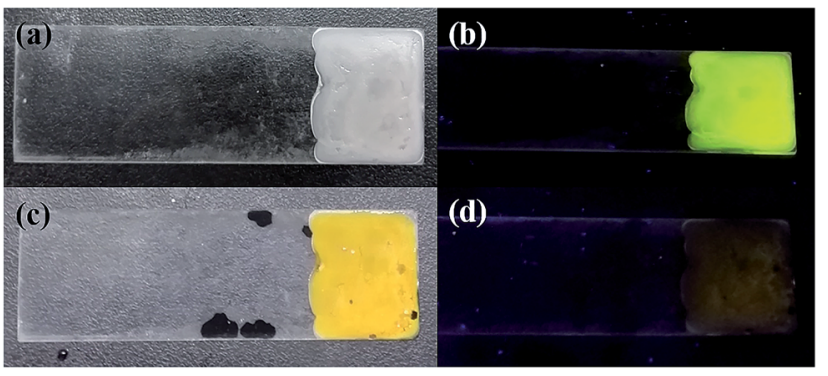

Fig. 7 (a) Glass plates coated with the gel film. (b) Fluorescence image of the glass plates coated with the gel film. (c) Film covered with AA bovine serum solution. (d) Fluorescence image of the film covered with AA bovine serum solution.

Notably, a series of other perylene fluorescent films for the detection of aniline vapor ${ }^{39,40}$ and phenol solutions ${ }^{\mathbf{4 1}}$ have been reported previously. However, these fluorescent perylene films are rarely applied for sensitive and portable AA detection. Considering these compounds with phenolic hydroxyl groups do not usually co-exist with AA, this visualized detection manner is also much more convenient for AA sensing, as well as the present PI-TiO gel system being more economical and easy to prepare.

\subsection{Model biological sample analysis}

The determinations of model biological samples with PI-TiO gel were performed by adding AA inside bovine serum with a standard addition technique. All serum samples were diluted $(10 \%)$ before the measurements. In order to ascertain the correctness of the results, AA was detected in the diluted samples at a final concentration of $150 \mu \mathrm{M}$. The results are displayed in Fig. 7. It was found that the PI-TiO film may be used for the direct determination of AA in real samples.

\begin{tabular}{|c|c|c|c|c|c|}
\hline Concentration & Phenol & Pyrocatechol & $\begin{array}{c}1,2,3- \\
\text { Benzenetriol }\end{array}$ & Glucose & $\begin{array}{c}\text { Tartaric } \\
\text { Acid }\end{array}$ \\
\hline \multicolumn{6}{|l|}{ 0 } \\
\hline & $(+)$ & $(+)$ & $(+)$ & $(+)$ & $(+)$ \\
\hline \multicolumn{6}{|l|}{$300 \mu \mathrm{M}$} \\
\hline & $(+)$ & $(-)$ & $(-)$ & $(+)$ & $(+)$ \\
\hline \multicolumn{6}{|l|}{$2 \mathrm{mM}$} \\
\hline & $(-)$ & $(-)$ & $(-)$ & $(+)$ & $(+)$ \\
\hline
\end{tabular}

Fig. 6 Fluorescence responses of the PI-TiO film to different hydroxyl-containing compounds at $300 \mu \mathrm{M}$ and $2 \mathrm{mM}((+)$ indicates fluorescence on, (-) indicates fluorescence off). 


\section{Conclusions}

In summary, a novel composite material, perylene carboxylatemodified inorganic TiO gel, has been prepared for sensitive and portable AA detection. By adjusting the reaction conditions, a strongly emitting PI-TiO gel can be obtained. During the detection process, inorganic TiO gel is not only used as a wet medium, but more importantly as a fluorescent quenching agent for the detection of AA. The film prepared on glass substrate can be applied as a simple visual colour and fluorescence double sensor for AA detection. Furthermore, the sensing principle and selectivity are carefully discussed and are ascribed to the strong coordination between phenolic hydroxyl groups and Ti(Iv). The portable ability of the sensor also has potential for human application in future point-ofcare testing.

\section{Conflicts of interest}

The authors declare no competing financial interest.

\section{Acknowledgements}

This work was supported by the National Natural Science Foundation of China (NSFC 21705106), the Program for Professor of Special Appointment (Eastern Scholar) at Shanghai Institutions of Higher Learning (No. TP2016023), Shanghai Sailing Program (17YF1405700) and Shanghai Natural Science Foundation (No. 18ZR1415400).

\section{References}

1 F. Y. Li, C. Y. Wang and W. W. Guo, Adv. Funct. Mater., 2018, 28, 1705876.

2 Y. Y. Hu, M. X. Xu, Y. R. Liu, X. Y. Xie, W. D. Bao, A. X. Song and J. C. Hao, J. Mater. Chem. A, 2017, 5, 1174-1181.

3 K. Q. Fan, X. B. Wang, Z. G. Yin, C. J. Jia, B. H. Zhang, L. M. Zhou and J. Song, J. Mater. Chem. C, 2018, 6, 1019210196.

4 P. Yang, H. S. Chen, J. P. Wang, Q. D. Che, Q. Ma, Y. Q. Cao and Y. N. Zhu, RSC Adv., 2014, 4, 20358-20363.

5 F. Ayari, M. Mhamdi, G. Delahay and A. Ghorbel, J. Porous Mater., 2010, 17, 265-274.

6 N. Mizoshita, T. Tani and S. Inagaki, Adv. Mater., 2012, 24, 3350-3355.

7 M. Schneider and K. Müllen, Chem. Mater., 2000, 12, 352362.

8 P. Wang, Y. Y. Wu, J. Wu, S. Wang, L. Yu, Q. Y. Zhu and J. Dai, J. Mater. Chem. C, 2013, 1, 7973-7978.

9 S. Y. Gan, L. J. Zhong, L. F. Gao, D. X. Han and L. Niu, J. Am. Chem. Soc., 2016, 138, 1490-1493.

10 B. Wang and C. Yu, Angew. Chem., Int. Ed., 2010, 49, 14851488.

11 S. H. Bai, L. L. Liang, C. L. Wang, H. F. Mehnane, C. H. Bu, S. J. You, Z. H. Yu, N. Cheng, H. Hu, W. Liu, S. S. Guo and X. Z. Zhao, J. Power Sources, 2015, 280, 430-434.
12 M. J. Robb, B. Newton, B. P. Fors and C. J. Hawker, J. Org. Chem., 2014, 79, 6360-6365.

13 Á. J. Jiménez, M. J. Lin, C. Burschka, J. Becker, V. Settels, B. Engels and F. Würthner, Chem. Sci., 2014, 5, 608-619.

14 Y. Avlasevich, C. Li and K. Müllen, J. Mater. Chem., 2010, 20, 3814-3826.

15 W. X. Liu, Z. G. Suo, Y. H. Liu, L. Y. Feng, B. B. Zhang, F. F. Xing and S. R. Zhu, Aust. J. Chem., 2019, 72, 206212.

16 S. J. Padayatty, A. Katz and Y. H. Wang, J. Am. Coll. Nutr., 2003, 22, 18-35.

17 R. Kanďár, P. Drábková and R. Hampl, J. Chromatogr. B: Anal. Technol. Biomed. Life Sci., 2011, 879, 2834-2839.

18 Y. Ma, M. G. Zhao, B. Cai, W. Wang, Z. Z. Ye and J. Y. Huang, Biosens. Bioelectron., 2014, 59, 384-388.

19 S. Z. Qureshi, A. Saeed, S. Haque and M. A. Khan, Talanta, 1991, 38, 637-639.

20 A. Khan, M. I. Khan, Z. Iqbal, Y. Shah, L. Ahmad, S. Nazir, D. G. Watson, J. A. Khan, F. Nasir, A. Khan and Ismail, Talanta, 2011, 84, 789-801.

21 X. Zhang, Y. C. Zhang and L. X. Ma, Sens. Actuators, B, 2016, 227, 488-496.

22 C. Q. Wang, J. Du, H. W. Wang, C. E. Zou, F. X. Jiang, P. Yang and Y. K. Du, Sens. Actuators, B, 2014, 204, 302-309.

23 J. Peng, J. Ling, X. Q. Zhang, L. Y. Zhang, Q. E. Cao and Z. T. Ding, Sens. Actuators, B, 2015, 221, 708-716.

24 P. K. Sukul, D. Asthana, P. Mukhopadhyay, D. Summa, L. Muccioli, C. Zannoni, D. Beljonne, A. E. Rowan and S. Malik, Chem. Commun., 2011, 47, 11858-11860.

25 F. Rigodanza, E. Tenori, A. Bonasera, Z. Syrgiannis and M. Prato, Eur. J. Org. Chem., 2015, 23, 5060-5063.

26 Y. Y. Wu, W. Luo, Y. H. Wang, Y. Y. Pu, X. Zhang, L. S. You, Q. Y. Zhu and J. Dai, Inorg. Chem., 2012, 51, 89828988.

27 L. Rozes and C. Sanchez, Chem. Soc. Rev., 2011, 40, 10061030.

28 W. S. Tung and W. A. Daoud, ACS Appl. Mater. Interfaces, 2009, 1, 2453-2461.

29 J. J. Han, A. D. Shaller, W. Wang and A. D. Q. Li, J. Am. Chem. Soc., 2008, 130, 6974-6982.

30 F. Schlosser, M. Moos, C. Lambert and F. Würthner, Adv. Mater., 2013, 25, 410-414.

31 Q. L. Zhang, L. C. Du, Y. X. Weng, L. Wang, H. Y. Chen and J. Q. Li, J. Phys. Chem. B, 2004, 108, 15077-15083.

32 P. Taheri, J. R. Flores, F. Hannour, J. H. W. D. Wit, H. Terryn and J. M. C. Mol, J. Phys. Chem. C, 2013, 117, 3374-3382.

33 N. Sikdar, D. Dutta, R. Haldar, T. Ray, A. Hazra, A. J. Bhattacharyya and T. K. Maji, J. Phys. Chem. C, 2016, 120, 13622-13629.

34 Z. M. Cheng, F. F. Xing, Y. L. Bai, Y. M. Zhao and S. R. Zhu, Asian J. Org. Chem., 2017, 6, 1612-1619.

35 A. P. Xagas, M. C. Bernard, A. H. Goff, N. Spyrellis, Z. Loizos and P. Falaras, J. Photochem. Photobiol., A, 2000, 132, 115120.

36 W. Jabs, W. Gaube, C. Fehl and R. Lukowski, Inorg. Chim. Acta, 1990, 175, 273. 
37 A. K. Dwivedi, M. Pandeeswar and T. Govindaraju, ACS Appl. Mater. Interfaces, 2014, 6, 21369-21379.

38 L. Zeininger, L. Portilla, M. Halik and A. Hirsch, Chem.-Eur. J., 2016, 22, 13506-13512.

39 C. Shang, G. Wang, M. He, X. Chang, J. Fan, K. Liu, H. Peng and Y. Fang, Sens. Actuators, B, 2017, 241, 13161323.
40 J. Fan, X. Chang, M. He, C. Shang, G. Wang, S. Yin, H. Peng and Y. Fang, ACS Appl. Mater. Interfaces, 2016, 8, 1858418592.

41 D. H. Zou, P. Wang, W. Luo, J. L. Hou, Q. Y. Zhu and J. Da, Inorg. Chem., 2018, 57, 1623-1629. 